\title{
EchoGéo
}

35 | 2016

Les découpages territoriaux en Europe

\section{Les découpages territoriaux en Europe}

Introduction

\section{Élisabeth Bonnet-Pineau et Christian Vandermotten}

\section{(2) OpenEdition}

Journals

Édition électronique

URL : https://journals.openedition.org/echogeo/14524

DOI : 10.4000/echogeo.14524

ISSN : 1963-1197

Éditeur

Pôle de recherche pour l'organisation et la diffusion de l'information géographique (CNRS UMR 8586)

Référence électronique

Élisabeth Bonnet-Pineau et Christian Vandermotten, « Les découpages territoriaux en Europe », EchoGéo [En ligne], 35 | 2016, mis en ligne le 19 avril 2016, consulté le 10 août 2021. URL : http:// journals.openedition.org/echogeo/14524; DOI : https://doi.org/10.4000/echogeo.14524

Ce document a été généré automatiquement le 10 août 2021.

EchoGéo est mis à disposition selon les termes de la licence Creative Commons Attribution - Pas d'Utilisation Commerciale - Pas de Modification 4.0 International (CC BY-NC-ND) 


\title{
Les découpages territoriaux en Europe
}

Introduction

\author{
Élisabeth Bonnet-Pineau et Christian Vandermotten
}

1 Aborder le champ d'étude des découpages territoriaux relève de la géographie politique et invite à analyser les rapports entre pouvoir et territoire comme des constructions politiques, sans ignorer que d'autres approches de la territorialité existent, tant le «paradigme territorial » est polysémique (M. Vanier, 2010).

2 Les articles réunis dans ce numéro abordent cette question sous deux angles d'attaque. Ils permettent d'établir des comparaisons entre les États de l'ouest de l'Europe et ceux de l'est, entrés dans l'Union en 2004, telle la Pologne, ou en 2007, telle la Bulgarie, lors de la dernière vague d'élargissement, et évidemment l'Allemagne, qui a dû relever le défi de l'unification et de la modernisation de l'ex-RDA.

Le premier angle d'attaque concerne la mise en place du maillage territorial dans le cadre de la formation des États-nations, et ses héritages contemporains.

4 L'État-nation s'est mis en place au XIX ${ }^{\mathrm{e}}$ siècle, en raison des besoins de la modernisation économique et politique dans le contexte du développement de marchés nationaux et de la rationalité héritée de l'esprit des Lumières. Il a mis en place des maillages territoriaux dont l'idéal était en cohérence avec les optima d'encadrement politique des territoires du début du XIXe siècle et qui étaient bien adaptés aux réalités économiques de cette époque, même s'ils furent bientôt quelque peu bousculés par le développement de l'industrie et du chemin de fer. Tout cela a été encadré par la production d'un cadre culturel et idéologique, fondé le plus souvent sur une langue commune, voire sur la construction d'une identité linguistique tentant d'éradiquer les dialectes et langues régionales. La formation de cet État-nation a souvent pu s'appuyer en Europe occidentale sur l'ancienneté et la pérennité du couple État/territoire. Ainsi, ce couple se construit en France de manière continue depuis le XIII ${ }^{e}$ siècle et la nation se prépare donc déjà de longue date dans le cadre de l'État royal, tout comme d'ailleurs en Angleterre. 
5 Dans ce contexte, l'archétype du découpage territorial, dans son uniformité et sa rationalité, correspondant à la formation d'États centralisés, est le département français. Produit de la Révolution, il est reproduit avec des nuances dans beaucoup de pays européens: provinces néerlandaises, belges, espagnoles, italiennes, etc. Le Royaume-Uni était proche du même modèle, même si le découpage en comtés était moins homogène et garda formellement plus de continuité avec les structures d'encadrement traditionnelles, tout comme la transition y fut plus précoce, plus longue et apparemment moins radicale qu'en France entre l'Ancien régime et l'État parlementaire.

6 La Belgique offre l'exemple particulier au cœur de l'Europe occidentale d'un État qui s'est construit tardivement, au XIX ${ }^{\mathrm{e}}$ siècle, un peu par le hasard de la reprise en main d'une révolte populaire par la bourgeoisie et d'un compromis entre les puissances voisines, sans base nationale évidente, même si la tentative fut faite - mais échoua - à en construire une, uniquement francophone. Par son histoire, par le rôle et la place des villes marchandes, la Belgique se rattache davantage à l'Europe médiane. La révolution industrielle a bénéficié à l'axe wallon tandis que la Flandre rurale et textile connaissait un déclin. Mais le glissement de l'industrie de la Wallonie vers la Flandre, amorcé entre les deux guerres, s'est amplifié après la Seconde Guerre mondiale, en inversant la dynamique de développement. Cette évolution a affaibli plus encore une construction nationale déjà mise à mal par les revendications flamandes qui se sont développées à partir de la fin du XIX ${ }^{\mathrm{e}}$ siècle, sur une base formellement culturelle, mais qui recouvrait tout autant des frustrations économiques. A partir de son héritage unitaire, la Belgique s'est trouvée emportée à partir des années 1960 dans un processus d'évolutions institutionnelles qui a abouti dès 1962 à l'introduction de découpages sur base linguistique, à tel point que la limite linguistique est devenue frontière intérieure intangible en 1962, et à un fédéralisme de méfiances en 1995, consacrant la dissolution de l'identité belge, qui n'avait jamais été pleinement réalisée de fait.

7 En Europe centrale, les héritages de l'encadrement territorial ancien furent mieux conservés, soit, comme en Suisse, que la division traditionnelle en cantons de tailles très variables n'ait pas été remise en question malgré la formation d'un État moderne après la guerre du Sonderbund, soit que la formation étatique fût tardive, précédée par le développement de l'idée nationale, et ait dû tenir compte de la force des pouvoirs locaux des princes ou des villes en adoptant une structure d'Empire fédéral : dès lors étaient conservés des découpages complexes hérités de l'Ancien régime jusqu'à la centralisation imposée par le national-socialisme. Dans les deux cas, la force des identités locales a conduit à des constructions fédérales, mais bien opposées à celles qui se sont mises en place récemment en Belgique, par décomposition de l'État unitaire. Dans ces structures fédérales, un strict partage prévaut entre les compétences de l'État et des régions, qui peut aller en Belgique jusqu'à une absence de hiérarchie entre l'État, les Régions (et les Communautés), chacun étant « souverain » pour les matières qui les concernent.

8 En Europe centre-orientale, la formation de l'État-nation ne s'est pas articulée avec les mêmes modèles d'essor économique précoce qu'en Europe occidentale, ce qui y a favorisé le développement d'idéologies nationales à forte base identitaire communautaire, comme d'ailleurs aussi en Allemagne, voire religieuses, celà dit sans pour autant prétendre que les nationalismes aient pu être moins vigoureux en France 
ou en Grande-Bretagne, même s'ils y étaient construits sur une base idéologique différente.

9 Ainsi, avant d'être un État et une forme territoriale, la nation polonaise s'est construite sur une culture, une "patrie spirituelle", faisant référence à un espace qui fut démembré, reconstitué, déplacé au gré des rapports de force. Le territoire polonais ne se laisse pas facilement saisir ni représenter à l'échelle historique. En effet, apparue au $\mathrm{X}^{\mathrm{e}}$ siècle, la Pologne a étendu son champ d'action loin vers l'est entre le XIV e et le XVIII ${ }^{e}$ siècle, avant de connaître un repli territorial après une série de partages au profit des grandes puissances limitrophes. La nation polonaise s'est formée autour d'une mémoire historique, d'une communauté de langues et de valeurs culturelles et religieuses. En 1945, remodelé dans sa configuration et homogénéisé dans sa composition ethnique, «le nouvel État polonais [a dû] construire son intégration territoriale dans un nouvel environnement géopolitique » (M. Foucher, 1993).

Sur le temps long, la Bulgarie contemporaine est quant à elle l'héritière revendiquée des empires bulgares qui se sont succédé du VII ${ }^{e}$ au XIV ${ }^{e}$ siècle. Après une domination ottomane, qui construisait l'encadrement politique autant sur la base des communautés religieuses que sur celle des territoires ${ }^{1}$, la Bulgarie moderne naît en 1878 , avec un territoire beaucoup plus restreint que celui dont elle ambitionnait de disposer, sur la base de ses fantasmes historiques.

11 La différence entre l'Europe occidentale et l'Europe centrale et orientale ne tient pas seulement aux conditions différentes de formation de l'État-nation et de ses fondements idéologiques, mais elle a aussi été renforcée par l'application à l'est, après la Seconde Guerre mondiale, du modèle spatial soviétique qui, imposant une centralisation de la gestion de toutes les activités économiques, a cherché à mettre le maillage administratif au service du contrôle de la mise en œuvre du plan économique dès lors en concordance avec lui. Toutefois, les régimes communistes n'ont pas appliqué un modèle unique de maillage administratif. Les héritages des maillages de l'avant-guerre ont parfois subsisté, mais pas partout. Les tentatives d'ajustement des découpages territoriaux au plan ont été multiples et les réformes territoriales furent donc d'abord des moyens privilégiés de diffusion du modèle économique planifié. Ainsi, dans cette partie de l'Europe, depuis 1945, la taille, le rôle des unités territoriales, le nombre de niveaux ont parfois été modifiés jusqu'à quatre ou cinq fois. En Bulgarie, en 1947, 1949, 1959, 1977 et 1988. En Pologne, en 1954, 1975 et 1983. Autrement dit, lorsque « le mur tombe ", on est loin d'être confronté à une page blanche.

Le second aspect traité concerne les modifications récentes des maillages territoriaux, censés devoir être adaptés aux réalités de la construction européenne, aux exigences de la compétition mondialisée des territoires et en même temps aux résurgences de sentiments identitaires régionaux face à des cadres nationaux qui apparaissent à certains moins incontournables que jadis dans ces contextes de modifications structurelles.

13 En effet, nombre de pays européens ont élaboré des réformes territoriales, qui visent à la fois des redécoupages et des décentralisations censés être mieux adaptés aux exigences européennes de cohésion territoriale et de compétition des territoires, quand ce n'est pas, comme en Espagne, en Écosse, ou plus encore en Belgique, pour répondre à des revendications identitaires qui profitent de l'affaiblissement des légitimités nationales (voir l'article de C. Vandermotten). D'autres réformes, au niveau le plus fin, tentent de répondre aux inadéquations de découpages municipaux hérités de sociétés 
où la mobilité était réduite, pour les rendre plus opérationnels dans un contexte d'urbanisation généralisée et de métropolisation, ainsi que de recours accrus aux services dans des cadres territoriaux élargis, qui imposent entre autres de nouveaux modèles de répartition de leur financement. Ainsi, en France, «la fabrique des territoires » apparaît comme le sport favori des élus nationaux, des savants, comme des experts (M. Vanier, 2010), alors que paradoxalement la rupture et la décentralisation des compétences y sont bien moins osées que dans d'autres pays, face à la centralisation et aux maillages territoriaux hérités. La dernière réforme y date de 2015, avec une promulgation de la loi en janvier 2016.

En Europe centre-orientale, ces exigences de redistribution des compétences viennent se superposer à des volontés de rompre avec les héritages du maillage de la période communiste, qui n'impliquent toutefois pas partout un véritable redécoupage territorial, comme le montre le cas bulgare, à la différence du cas polonais. La rupture a évidemment été très marquée en ex-RDA, qui devait radicalement s'affranchir des marquages spatiaux hérités de l'époque socialiste, en retrouvant la structure fédérale de la RFA qui avait été bannie en 1952 au profit d'une forte centralisation. La réunification des deux Allemagnes et l'application à l'est des mêmes maillages territoriaux qu'à l'ouest n'empêche évidemment pas une forme de «mezzogiornisation » de l'est, même si l'objectif d'équivalence des conditions de vie est énoncé dans la Constitution. Pourtant, cette quête d'équilibre territorial représente un enjeu considérable dans un pays marqué par des identités historiquement fortes, voire des traditions d'indépendance régionale, particulièrement sensibles dans les zones qui ne relevaient pas de la Prusse mais de leurs propres rois, comme la Saxe ou la Bavière.

De manière générale, le premier niveau de découpage territorial tend à se renforcer Sur le moyen terme, celui des dernières décennies, il convient d'ailleurs souvent, avant même de parler de renforcement de ce niveau, tel qu'il est entendu aujourd'hui, d'évoquer sa création ex nihilo. À la sortie de la dernière guerre, la trame de premier niveau dans les États européens unitaires correspondait à une maille homogène de l'ordre de celle du département français. Les régions de plus grande taille, et parfois de dimensions moins homogènes, vont progressivement se superposer à ce niveau en Italie, en commençant à partir de 1946 par les régions à statut spécial, en France, en Belgique, en Espagne, après la fin du franquisme, en Pologne. En Allemagne, le retour à la démocratie après la Seconde Guerre mondiale reconstitue une organisation en Länder, qui avait été de fait sinon de droit supplantée durant le régime nazi par la trame homogène des Gaue, mise en place à partir de 1934.

Cette tendance à la création d'entités de premier niveau de taille généralement plus grande, puis à leur renforcement, d'ampleur plus ou moins marquée selon les pays, renvoie à divers objectifs. Dans certains cas, elle s'appuie, ou tente de désamorcer, des revendications identitaires dont la légitimité politique s'est affirmée, comme en Espagne, en Italie, au Royaume-Uni, en Belgique, sans parler de l'Allemagne où la réalité régionale n'a été brimée que durant la période national-socialiste (et en ex$\mathrm{RDA}$ ). Plus récemment, il s'est agi de rencontrer les exigences des politiques européennes de cohésion territoriale, qui ont renforcé les politiques plus anciennes d'aide régionale et affichent, depuis 1999, l'objectif de favoriser la convergence en réduisant les écarts de développement entre les États et les régions de l'Union. Enfin, 
chaque grande métropole veut s'imposer dans la compétition internationale des territoires en encadrant et appuyant le développement d'un espace élargi.

Ce « grand niveau 1 » va de soi en Allemagne, où il peut non seulement s'appuyer sur les fortes identités des Länder, mais aussi sur une structuration polynucléaire et une polarisation régionale étayée sur les « Metropolregionen » ou régions métropolitaines réparties de manière équilibrée sur l'ensemble du territoire, même si persiste la dissymétrie est-ouest. Son implantation à l'est relevait de l'évidence.

Son renforcement en Italie peut aussi s'appuyer sur un réseau de villes finement maillé, même si la dualité nord-sud reste lisible quand on confronte Milan et Rome, pour ne pas parler de Naples ou de Palerme. Dès 1948, l'Italie s'est définie comme un État régionalisé pour "reconstruire la démocratie passant par le territoire " (D. Rivière, 2014). La démocratie-chrétienne ayant exercé les responsabilités à tous les échelons jusqu'en 1980, ce n'est qu'en 1990 que l'Italie put renforcer les pouvoirs régionaux, communaux et métropolitains, dont la légitimité apparaissait renforcée par la faillite de l'État. La montée en puissance des régions est corollaire à l'approfondissement de la décentralisation. Elle s'appuie sur des traditions identitaires régionales qui ont pu persister du fait d'une unification tardive, voire se nourrir des inégalités régionales criantes. Toutefois, la création de métropoles en 2014-2015 participe d'une réaction de l'État central, qui met les régions au pas et cantonne le Sénat, qui les représentait, à un rôle secondaire (voir le texte de D. Rivière).

En Espagne, l'affirmation des identités régionales a été libérée par la fin du franquisme, allant jusqu'aux revendications indépendantistes en Catalogne et au pays Basque.

Une telle affirmation a conduit à un fédéralisme "de séparation » en Belgique, qui n'existe sans doute plus que parce que sa capitale est au cœur du fonctionnement économique de l'ensemble du pays, tout en étant étranglée par une délimitation étroite, qui exclut de sa gestion une grande partie de son aire métropolitaine.

La France aussi a voulu renforcer la reconnaissance du niveau régional pour mieux s'inscrire dans les normes européennes, qui visent à consolider le couple régionEurope, mais aussi pour compenser une tendance générale au repli de l'État en matière d'aménagement et de développement des territoires dans un contexte général plus néo-libéral. Mais elle semble s'être arrêtée au milieu du gué. Le chantier ouvert de la décentralisation conduit par l'État en 1982, poursuivi en 2004, relève plus du délestage de l'État central lors des transferts de compétences et de charges aux niveaux locaux que de la redéfinition des principes d'articulation et de coordination entre niveaux de gestion, entre fonctions politiques et administratives (voir le texte d'E. Bonnet-Pineau).

La réforme régionale (loi du 16 janvier 2015) vise à doter la France d'une nouvelle architecture territoriale, consistant à créer de grandes régions pour bénéficier de gains de compétitivité. Elle devait aussi clarifier les compétences entre les collectivités territoriales. Or, si la réduction du nombre de régions était relativement facile à conduire (sans que le gain d'efficacité qui devait en résulter ait été réellement démontré), la suppression des départements n'est pas chose aisée ; il reste un échelon de proximité et de traitement de la solidarité nationale. La refonte de la carte régionale s'est d'ailleurs faite par fusion des régions existantes, sans modification des départements qui les composent.

24 La revue des missions et de la déconcentration des pouvoirs n'a pas non plus débouché sur une réorganisation des compétences, ni sur une augmentation de la capacité 
budgétaire et de décision politique des régions. Le partage des rôles entre différents échelons régi par une répartition des domaines d'intervention s'inscrit en contradiction avec le principe du développement territorial, lequel invite à intervenir dans tous les champs sectoriels. Enfin, la non-définition des conditions financières permettant de réaliser une telle action et $d$ 'en assurer la pérennité fait peser de nombreuses incertitudes sur la réussite de la réforme au regard des objectifs qui ont été énoncés.

La loi de modernisation de l'action publique territoriale et d'affirmation des métropoles du 27 janvier 2014 a institué les métropoles de Paris, Lyon et Marseille et précisé les modalités de mise en œuvre d'une nouvelle gouvernance métropolitaine. Elle invite à poser la question de la place de l'État dans la gouvernance métropolitaine et celle des relations entre métropoles et régions. Construction compliquée dans le cas de Paris, dont les enjeux sont politiques tout autant qu'économiques et financiers. Paris-capitale doit réussir à composer avec l'État et la Région Île-de-France.

Il est dommage que ce numéro ne contienne pas d'article relatif au Royaume-Uni, qui reste en fin de compte le pays le plus centralisé d'Europe, avec un niveau régional inconsistant, du moins si on ne considère pas comme tel les dévolutions de pouvoir dont disposent l'Écosse, le pays de Galles et l'Irlande du nord.

Cette question des rapports entre la ville-capitale et le reste du territoire est aussi particulièrement aiguë dans les pays d'Europe centre-orientale où, à l'heure de la mondialisation et en dépit de l'homogénéisation que les régimes socialistes avaient imposé à l'armature urbaine de premier niveau, les régions les mieux connectées à l'espace européen, celles qui gagnent et qui accroissent leur différentiel de développement avec le reste du pays, sont précisément les régions-capitales. C'est le cas en Pologne avec Varsovie, et plus encore en Bulgarie avec Sofia.

En Europe centre-orientale (sauf en ex-RDA), malgré les réformes du maillage territorial et la volonté affichée de renforcer les compétences des régions, l'affirmation du premier niveau reste encore fragile, faute de traditions de décentralisation, même avant les régimes communistes, et eu égard aux méfiances d'États de formation récente, visant avant tout à affirmer leurs frontières. En Pologne, la réduction du nombre de voïvodies à 16 en 1999 s'inscrit dans une volonté de renforcer ce premier niveau. Mais en Bulgarie, le rôle de la région reste inconsistant, ce qui tient à la faiblesse des grandes villes régionales, à l'absence de régions historiques et à la limitation des compétences des régions à la seule coordination des intérêts locaux et nationaux, même si elles contrôlent l'échelon communal (voir le texte d'E. Boulineau). Les régions y ont surtout été créées pour satisfaire aux exigences statistiques d'EUROSTAT, mais de fait la manne financière européenne n'est parvenue qu'à l'échelon central, avant suspension pour fraude et corruption.

\section{Le niveau intermédiaire}

Dans la plupart des pays européens, sauf les plus petits d'entre eux, il existe un échelon intermédiaire entre celui du découpage de premier niveau et le niveau local.

Le niveau intermédiaire pèse très peu dans les États fédéraux. C'est le cas en Allemagne pour le Regierungsbezirk, qui n'existe d'ailleurs que dans les plus grands Länder. En Belgique, les compétences du niveau provincial se sont affaiblies, plus encore en Wallonie qu'en Flandre, et Bruxelles y échappe aujourd'hui. Il n'y a pas de niveau intermédiaire en Suisse. 

européenne (voir le texte de J.-B. Grison), permet de souligner la position singulière de la France, en raison du nombre de très petites communes, issues du découpage paroissial sous l'Ancien régime, et de la résistance de celles-ci aux processus de fusion. Pourtant l'autonomie des communes face au pouvoir central est de fait moindre que dans d'autres pays. L'attachement des Français à ces mailles de proximité justifie la force d'inertie dont elles font preuve et leur longévité. La généralisation de l'intercommunalité est apparue comme le compromis permettant de ne pas affronter la résistance des communes, et de leurs élus, tout en se dotant de structures de taille suffisante pour assurer et rationaliser les services aux populations. On peut y ajouter récemment des incitations financières à la fusion des plus petites communes.

39 françaises, n'ont pas résisté à un processus général de fusion en 1976, alors que pourtant l'autonomie des pouvoirs locaux est plus forte. À la différence de la France aussi, le maillage local n'est pas partout de la compétence de l'État: ainsi en Belgique, la trame communale et la tutelle des communes sont maintenant de la compétence des Régions, tout comme elles le sont de celle des Länder en Allemagne: de la sorte cette 
trame y a été plus ou moins modifiée selon les Länder ; l'émiettement a été conservé en Rhénanie-Palatinat, alors qu'en revanche les fusions ont été radicales en Rhénanie du Nord - Westphalie; en Saxe, le Land le plus urbain de l'ex-RDA, les modifications du périmètre des communes visant une rationalisation de l'organisation du territoire au bénéfice des lieux centraux ont suivi les mêmes voies qu'en Rhénanie du Nord Westphalie (voir le texte de D. Florentin).

En Angleterre la centralisation exclut toute consistance du niveau régional. La complexité et la non-homogénéité du maillage local dans ce pays, ainsi que la multiplication d'agences gouvernementales, y traduisent la mainmise $d u$ gouvernement central, renforcée encore par les gouvernements conservateurs qui trouvent dans les grandes villes les principaux foyers de leur opposition politique. Pays paradoxal, où la diversité des structures masque la centralisation, alors que dans les pays continentaux qui furent peu ou prou influencés par la France, celle-ci fut au contraire imposée à travers leur homogénéisation.

41 L'élément le plus marquant des mutations en Europe centre-orientale est sans doute le retour à une autonomie des pouvoirs locaux, comme en Pologne suite aux réformes de 1990, 1999 et 2011 sur l'autonomie territoriale. La réforme de 1999 en Bulgarie, considérée comme une "pseudo-réforme", a été complétée en 2007 par une modification de la Constitution introduisant plus d'autonomie fiscale en faveur des communes. L'introduction de nouveaux modes de financement, décentralisés, des pouvoirs locaux peut à son tour impliquer des exigences de fusion des entités locales, comme le montre l'exemple de la Saxe, alors que, paradoxalement, si les municipalités avaient perdu leur autonomie dans les pays d'Europe centre-orientale sous la période communiste, le découpage de la trame locale s'est maintenu assez stable.

Pour conclure, il reste que s'il existe incontestablement une dialectique et des interactions entre modalités de gestion et maillage politico-administratif des territoires d'une part, production de l'espace, dans sa globalité économique, sociale et culturelle d'autre part, sans pour autant qu'il y ait toujours adéquation parfaite entre ces deux dimensions, qui ont en outre leurs temporalités propres, il n'empêche que le politique a parfois tendance à surestimer l'impact réel des réorganisations territoriales sur les devenirs des espaces. La punition infligée à Lyon par les Révolutionnaires jacobins en ne lui attribuant que la préfecture d'un département largement amputé n'a pas empêché que la ville devienne la deuxième métropole française. Hors d'Europe, des constructions ex-nihilo de nouvelles capitales n'ont généralement pas détrôné les grandes métropoles, qui étaient les capitales historiques et restent les principaux foyers économiques. La métropolisation d'un espace central belge autour de Bruxelles se poursuit malgré des décisions politiques qui visaient ouvertement et visent toujours à contenir la capitale du pays dans un carcan, même si ces décisions rendent plus difficile la gestion de ce territoire (C. Vandermotten). En fait, la volonté de rationaliser la gestion des territoires, de les réorganiser sur des canevas supposés les rendre plus compétitifs, plus efficients, mieux aptes à rencontrer les contraintes financières dans le contexte de la mondialisation procède d'un postulat attribuant aux territoires euxmêmes un pouvoir de régulation et d'impulsion, qu'ils ne détiennent pas en tant que tels, mais qui est soumis à la conjonction de l'inertie de structures héritées et de multiples décisions d'acteurs dont les stratégies sont loin de répondre aux seuls desiderata politiques. 


\section{BIBLIOGRAPHIE}

Foucher M., 1993. Fragments d'Europe. Fayard.

Rivière D., 2014. L'Italie de la crise. Les cafés Géo (www.cafe-geo.net/litalie-de-la-crise)

Vanier M., 2002. Recomposition territoriale française : la voie française. L'information géographique, p. 99-112.

\section{NOTES}

1. Idée que l'on retrouve, sous une forme modernisée et «laïcisée », dans les projets des austromarxistes d'avant la Première Guerre mondiale quant à une organisation fédérale de l'Empire austro-hongrois fondée sur l'autonomie des communautés ethniques plutôt que sur celle de territoires.

\section{INDEX}

Thèmes : Sur le Champ - Sur le Terrain

\section{AUTEURS}

\section{ÉLISABETH BONNET-PINEAU}

Elisabeth Bonnet Pineau, danieloster@wanadoo.fr, est géographe et consultante.

\section{CHRISTIAN VANDERMOTTEN}

Christian Vandermotten cvdmotte@ulb.ac.be, est Professeur ordinaire à l'Université Libre de Bruxelles et membre de l'Académie Royale des Sciences, des Lettres et des Beaux-Arts de Belgique. 